Conclusion There have been distinct inequalities in the reduction of spending for CEP services. LAs with a higher baseline level of deprivation, those with a single-tier local government structure, and English rural LAs have been worst affected. These inequalities in cuts to services that impact public health risk widening geographical and social health inequalities. Understanding these inequalities will provide crucial evidence to inform the UK government's 'levelling up' strategy as the country recovers from the COVID19 pandemic. One limitation of our study is that we were unable to investigate how resources have been distributed within LAs.

\section{P88 HOW CAN WE OPTIMISE THE CO-LOCATION OF WELFARE RIGHTS ADVICE IN A HEALTH SETTING TO BENEFIT THOSE MOST IN NEED? A NARRATIVE SYNTHESIS SYSTEMATIC REVIEW}

${ }^{1}$ Sian Reece*, ${ }^{2}$ Josie Dickerson, ${ }^{3}$ Kate Pickett. ${ }^{1}$ Health Sciences, Hull York Medical School, York, UK; ${ }^{2}$ Bradford Institute for Health Research, Bradford Teaching Hospitals NHS Foundation Trust, Bradford, UK; ${ }^{3}$ Department of Health Sciences, University of York, York, UK

\subsection{6/jech-2021-SSMabstracts. 174}

Background Financial difficulties can precipitate and perpetuate mental health problems and are a predictor of chronic physical illness. The COVID-19 pandemic is an unprecedented public health crisis with profound health and socioeconomic impacts and the long-term consequences are yet to be seen. The poorest and most vulnerable groups are worst affected, further widening the health inequality gap. It is known that there is low uptake of universal and means-tested benefits in some communities. Various schemes have been put in place to improve uptake of benefits by co-locating welfare advice within health services. However, we need more research on how to do this most effectively to reach the populations most in need and to assess its impact on health, social and financial outcomes.

Methods We conducted a critical systematic narrative synthesis review of relevant papers published between 2010-2020 using an evidence-led framework described by Rodgers et al. consisting of four elements used to characterise the approach: developing a theory; developing a preliminary synthesis; exploring relationships within and between studies; and assessing the robustness of the evidence.

Results In total, 16,625 participants accessed and were supported by the welfare services, with $£ 19,576,223$ successfully claimed as one-off payments for participants. Participants benefitted from an additional $£ 2,757$ household income per annum and improved financial literacy. The services across this review generated an average of $£ 21.95$ of social, economic and environmental return on investment per $£ 1$ invested. Co-located welfare advice services actively incorporate elements of proportionate universalism and target those who are most at need of this support and who would not otherwise access the services. The services raised the profile of the importance and value of addressing social determinants of health with healthcare professionals and policy makers and de-stigmatising access to welfare services and being in receipt of benefits. Access to welfare services also produced demonstratable cost savings for the NHS. Welfare services facilitated more appropriate use of NHS resources, promoting access for those who needed it but were not accessing it and reducing the burden of welfare issues on healthcare practitioner's time.

Discussion Overall, this review demonstrates significant financial gains for participants and for the first time demonstrates wider welfare benefits to participants, including access to housing, food, transport and employment. This contributes to the theory that these welfare services both directly and indirectly address social determinants of health thereby improving health and wellbeing and reducing health inequalities.

\section{P89 MEASURING THE IMPACT OF A MEDIA CAMPAIGN ON APPLICATIONS FOR LEGAL AID TO REGISTER A POWER OF ATTORNEY; AN INTERRUPTED CONTROLLED TIME SERIES}

Kate Levin*, Jill Carson. Public Health Directorate, NHS GGC, Glasgow, UK

\subsection{6/jech-2021-SSMabstracts. 175}

Background Power of attorney (POA) is a legal document in which one individual gives authority to another to act or make welfare and health care decisions for them, in the event that they become incapacitated. A public awareness campaign, first implemented in Glasgow City, and rolled out to other parts of Scotland in stages, was previously found to increase POA registrations. The current study measures the impact of the campaign on registrations and applications to legal assistance, as a proxy for low socioeconomic (SES) status applications.

Methods POA registration and legal aid application (LAA) for POA data were analysed between January 2010 and December 2018. Multilevel Negative Binomial models for POA and LAA registrations nested by council and annual quarter were run using RIGLS estimation in MLwin, adjusting for pre and post intervention period for each locality, campaign (variable ranging between 0-3 dependent on intensity of campaign measured by the number of media platforms received), and offset term mid-year population estimate for those aged 65 year + . A further model was then run for outcome LAA registrations with offset term total registrations, in a similar way, to examine the impact of the campaign on the proportion of registrations with legal assistance.

Results In Glasgow City POA registrations rose by $33.3 \%$ between 2013 and 2014, following the introduction of the campaign, compared with $17.3 \%$ in the rest of Scotland. LAA during this period rose by only $10.6 \%$ in Glasgow and $16.3 \%$ in the rest of Scotland. However, when the data for the whole study period were modelled, the relative risk of a registration for those living in an area with the full campaign was $R R=1.12(1.07,1.17)$ those living where no campaign was in place. Relative Risk rose in an approximate stepwise fashion with increasing campaign intensity. Relative risk of a LAA for the same group was $1.10(1.01,1.21)$. When LAA proportion of registrations was instead modelled over time, ie LAA as outcome with total registrations as an offset, the campaign variable was not significantly associated with the outcome.

Conclusion During the period of the campaign, area-level increases in LAA were associated with the timing, intensity and location of the media campaign, in a similar way to that of all POA registrations. This suggests that the campaign 
increased registrations from both lower socioeconomic backgrounds and all other SES in a similar way, therefore neither reducing nor increasing inequalities in POA registrations.

\section{\begin{tabular}{l|l} 
P90 IMPLEMENTATION OF STRATEGIES FOR FOSTERING \\
\hline
\end{tabular} STRENGTHS-BASED ADULT SOCIAL WORK IN THE UK: A SYSTEMATIC REVIEW OF RESEARCH EVIDENCE}

${ }^{1}$ Anna Price* ${ }^{1}$ Latika Ahuja, ${ }^{1}$ Charlotte Bramwell, ${ }^{1}$ Simon Briscoe, ${ }^{1}$ Liz Shaw, ${ }^{1}$ Michael Nunns, ${ }^{2}$ Gareth O'Rouke, ${ }^{3}$ Samantha Baron, ${ }^{1}$ Rob Anderson. ${ }^{1} H S D R$ Evidence Synthesis Centre, University of Exeter Medical School, Exeter, UK; ${ }^{2}$ Institute of Health Research, University of Exeter Medical School, Exeter, UK; ${ }^{3}$ Department of Social Care and Social Work, Manchester Metropolitan University, Manchester, UK

\subsection{6/jech-2021-SSMabstracts. 176}

Background A 'strengths-based approach' focusses on peoples' goals and resources rather than their problems. Social care professionals and organisations are striving to practise in a strengths-based way, especially since the Care Act of 2014. However, challenges remain in implementing strengths-based approaches into practise, and uncertainty remains about their effectiveness. This systematic review aimed to summarise research evidence on the effectiveness and the implementation of different strengths-based approaches within adult social work in the UK.

Methods We searched seven databases: MEDLINE ALL, PsycINFO, Social Policy and Practice, HMIC, CINAHL, ASSIA and the Campbell Library. Supplementary web searches were conducted. No date or language limits were used. Eligible studies were about adults ( $\geq 18$ years) being supported or assessed by social workers; or initiatives involving adult social care teams. For the effectiveness question, outcomes could be directly related to individual outcomes or outcomes at the level of families or communities. The Cochrane Risk of Bias Tool was chosen to appraise the quality of effectiveness studies, and qualitative implementation studies were assessed using the Wallace criteria. Findings were tabulated and analysed using framework synthesis. Studies that were not synthesised were summarised descriptively.

Results Of 5,030 studies screened, none met our inclusion criteria for the effectiveness question. Fifteen qualitative or mixed methods studies met criteria for the implementation question, six were assessed as 'good quality'. Seven examined Making Safeguarding Personal (MSP) and the remaining eight studies examined Local Area Coordination, Solution Focused Therapy, Family Group Conferencing, Asset-based Community Development, Strengths-based with Relationship-based Approach, Asset-based approaches, and Motivational Interviewing. Studies on Making Safeguarding Personal (MSP), were synthesised into the following themes of implementation factors: 1) MSP as an intervention: seen as initially demanding but with long-term advantages. 2) Culture and Settings: required broad cultural changes; 'outward facing' and smaller/specialist councils tended to find this easier. 3) Individual characteristics: related to enhancing the knowledge, skills and confidence of practitioner and stakeholders in MSP; and service user willingness to engage. 4) Embedding and sustaining MSP: depended on strong leadership and active engagement at all levels. For the remaining eight studies of seven strengths-based approaches, we provide a summary of findings.

Discussion There is a lack of good quality research evidence evaluating the effectiveness or implementation of strengths- based approaches. The synthesis revealed a wide range of factors that enabled or inhibited successful implementation of Making Safeguarding Personal. These may have wider relevance for implementation of other strengths-based models of social work practice.

\section{P91 SENSITIVITY ANALYSES FOR UNMEASURED CONFOUNDING: RECOMMENDATIONS FOR POPULATION HEALTH RESEARCH}

Anna Pearce*, Vittal Katikireddi, Alastair Leyland, Ruth Dundas. MRC/CSO Social and Public Health Sciences Unit, University of Glasgow, Glasgow, UK

10.1136/jech-2021-SSMabstracts. 177

Background Many population health research questions rely on observational data, where unmeasured confounding is a major source of bias. Sensitivity analyses for unmeasured confounding are increasingly applied, but often without sufficient consistency and transparency. We propose accessible recommendations to guide applied researchers in using two existing sensitivity analyses. 1) Bias Factor (BF), which is derived from the expected strength of associations between unmeasured confounder and exposure/outcome, based on expert knowledge and previous research. The main effect estimate (and confidence intervals, CIs) are adjusted using the BF. 2) E-value (EV), which identifies the strength of associations between unmeasured confounder and exposure/outcome required to entirely attenuate the main effect estimate (or for CIs to contain the null)

Methods We conducted a scoping review for commentaries and reviews discussing the application, strengths, and limitations of the BF and EV. We triangulated these with epidemiological guidance (e.g. STROBE) and informal discussions with quantitative researchers in applied statistics, epidemiology and social policy.

Results The BF was criticised for the potential for authors to selectively pick confounder associations that minimally impact the results. The EV removes the potential for author bias and future-proofs analyses (as knowledge of confounders advances). However, it potentially discourages authors' rigorous and transparent consideration of unmeasured confounding; and places burden upon the reader to judge whether this degree of confounding would seem feasible. Furthermore, population research typically aims to estimate an effect size (not merely the existence of an effect, which is the focus of the EV). Initial recommendations. Unmeasured confounders are identified at protocol stage. A range of exposure/outcome associations are identified for the confounder(s), from systematic reviews, high-quality individual studies, and expert opinion. At publication stage: 1) the full range of BFs are applied to the main effect size and CIs, reported in full, and the most pertinent highlighted in the discussion; 2) The EV, for the main effect and CIs, is compared with best estimates derived using the $\mathrm{BF}$, observed confounders-exposure/outcome associations, and effect sizes for other important exposure/ outcome risk factors; 3) The importance of the effect size after considering potential residual confounding should be assessed; 4) Results are discussed in context of other threats to bias, including measurement error among measured confounders (as applied in primary studies and systematic reviews). 